\title{
Stability of Drinking Reductions and Long-term Functioning Among Patients with Alcohol Use Disorder
}

\author{
Katie Witkiewitz, $P h D^{7}$ (D) , Henry R. Kranzler, $M D^{2}$, Kevin A. Hallgren, $P h D^{3}$, \\ Deborah S. Hasin, PhD' 4 , Arnie P. Aldridge, PhD ${ }^{5}$, Gary A. Zarkin, PhD ${ }^{5}$, Karl F. Mann, MD 6 , \\ Stephanie S. O'Malley, $P h D^{7}$, and Raymond F. Anton, $M D^{8}$
}

\begin{abstract}
'Department of Psychology, University of New Mexico, MSC 03-2220, Albuquerque, NM, USA; '² Department of Psychiatry, University of Pennsylvania, Philadelphia, PA, USA; ${ }^{3}$ Department of Psychiatry and Behavioral Sciences, University of Washington, Seattle, WA, USA; ${ }^{4}$ Department of Epidemiology, Columbia University, New York, NY, USA; ${ }^{5}$ Behavioral Health Research Division, RTI International, Research Triangle Park, NC, USA; ${ }^{6}$ Central Institute of Mental Health, Medical Faculty Mannheim, Heidelberg University, Mannheim, Germany; ${ }^{7}$ Yale School of Medicine, New Haven, CT, USA; ${ }^{8}$ Department of Psychiatry and Behavioral Sciences, Medical University of South Carolina, Charleston, SC, USA.
\end{abstract}

BACKGROUND: The World Health Organization (WHO) categorizes alcohol consumption according to grams consumed into low-, medium-, high-, and very-high-risk drinking levels (RDLs). Although abstinence has been considered the ideal outcome of alcohol treatment, reductions in WHO RDLs have been proposed as primary outcomes for alcohol use disorder (AUD) trials.

OBJECTIVE: The current study examines the stability of WHO RDL reductions and the association between RDL reductions and long-term functioning for up to 3 years following treatment.

DESIGN AND PARTICIPANTS: Secondary data analysis of patients with AUD enrolled in the COMBINE Study and Project MATCH, two multi-site, randomized AUD clinical trials, who were followed for up to 3 years post-treatment (COMBINE: $n=694$; MATCH: $n=806$ ).

MEASURES: Alcohol use was measured via calendarbased methods. We estimated all models in the total sample and among participants who did not achieve abstinence during treatment.

KEY RESULTS: One-level RDL reductions were achieved by $84 \%$ of patients at the end of treatment, with $84.9 \%$ of those individuals maintaining that reduction at a 3-year follow-up. Two-level RDL reductions were achieved by $68 \%$ of patients at the end of treatment, with $77.7 \%$ of those individuals maintaining that reduction at a 3-year follow-up. One- and two-level RDL reductions at the end of treatment were associated with significantly better mental health, quality of life (including physical quality of life), and fewer drinking consequences 3 years after treatment $(p<0.05)$, as compared to no change or increased drinking.

CONCLUSION: AUD patients can maintain WHO RDL reductions for up to 3 years after treatment. Patients who had WHO RDL reductions functioned significantly

Prior Presentations Preliminary results from these analyses were presented at the Lisbon Addictions meeting in Lisbon, Portugal, on October 23, 2019.

Supplementary Information The online version contains supplementary material available at https://doi.org/10.1007/s11606-020-06331-x.

Received January 2, 2020

Accepted October 18, 2020

Published online November 12, 2020 better than those who did not reduce their drinking. These findings are consistent with prior reports suggesting that drinking reductions, short of abstinence, yield meaningful improvements in patient health, well-being, and functioning.

KEY WORDS: World Health Organization risk drinking levels; alcohol use disorder; reduced alcohol consumption; alcohol treatment outcomes; lowrisk drinking; alcohol dependence.

J Gen Intern Med 36(2):404-12 DOI: $10.1007 / \mathrm{s} 11606-020-06331-\mathrm{x}$

(C) The Author(s) 2020

\section{INTRODUCTION}

Alcohol use disorder (AUD) is highly prevalent and has high social and economic costs, ${ }^{1}$ yet most individuals with AUD never seek treatment. ${ }^{2}$ A primary reason reported by individuals with AUD for not seeking treatment is that they were not ready to stop drinking. ${ }^{2}$ Thus, a commitment to a goal of abstinence is an extremely high criterion for engaging individuals in treatment for AUD and determining whether alcohol treatment is successful. ${ }^{3}$ Drinking reductions, short of abstinence, are associated with considerable improvements in both health and functioning in general population and treatment samples. ${ }^{4-9}$ Broadening the definition of "success" in AUD treatment to include reduced drinking could decrease the public health burden of AUD by engaging more individuals in treatment for the disorder, ${ }^{3,10}$ and would provide more options for clinicians to offer patients, beyond recommending abstinence from alcohol.

\section{Defining "Success" in AUD Treatment}

The Food and Drug Administration ${ }^{11}$ recommends sustained abstinence or no heavy drinking days (defined as more than 3 drinks in a day for women and 4 drinks in a day for men) as indicators of success for AUD treatment. The European Medicines Agency ${ }^{12}$ (EMA) recommends abstinence, but also considers reduced drinking as a successful intermediate outcome. The EMA outcome includes reductions in total alcohol 
consumption, heavy drinking days, or World Health Organization (WHO) risk drinking levels (RDLs), ${ }^{13}$ which are defined by the number of grams $(\mathrm{g})$ of alcohol consumed per day, including abstinent (0 g males/females), low risk (1 to $40 \mathrm{~g}$ males/1 to $20 \mathrm{~g}$ females), medium risk (41 to $60 \mathrm{~g}$ males/ 21 to $40 \mathrm{~g}$ females), high risk (61 to $100 \mathrm{~g}$ males/41 to $60 \mathrm{~g}$ females), or very high risk $(101+\mathrm{g}$ males $/ 61+\mathrm{g}$ females $)$. The WHO RDLs provide concrete guidance for defining levels of drinking (see Fig. 1 for daily and weekly US approximate standard drink equivalents) associated with specific levels of risk and, relatedly, offer targets for reducing drinking (e.g., a two-level reduction from very high risk to medium risk). Recent studies have shown that reductions in WHO RDLs are associated with improvements in health and functioning among population samples ${ }^{5,6,8}$ and individuals in AUD treatment. $^{4,7,9}$

\section{Current Study}

Abstinence has long been considered a stable outcome for individuals with AUD, ${ }^{14,15}$ and numerous studies have questioned whether "controlled" drinking can be maintained. $^{15-17}$ The debate over whether controlled drinking is achievable has been considered in the academic literature for nearly 50 years, ${ }^{18}$ but concrete guidance has not emerged on the magnitude of the reduction that is necessary for sustained health benefits. More recent work has shown that drinking reductions, as assessed by the WHO RDLs, are often seen over the first post-treatment year, ${ }^{7}$ but whether these reductions can be maintained for longer periods and whether WHO RDL reductions, short of abstinence, are associated with sustained improvements in functioning remain unclear. Given the common perception that individuals with severe AUD have difficulty sustaining reduced drinking, ${ }^{3,19}$ it is also unknown whether the severity of AUD at baseline moderates these effects. To address these gaps in the literature, we examined whether WHO RDL reductions of at least one level (e.g., very high to high) or at least two levels (e.g., very high to medium) can be maintained and whether these reductions in drinking are associated with long-term improvements in functioning. We hypothesized that one- and two-level reductions in WHO RDLs, even among individuals who did not achieve absti- nence, would be maintained and associated with better mental health, quality of life, and functioning 3 years following treatment.

\section{METHODS}

\section{Participants and Procedures}

We used data from the COMBINE Study ${ }^{20}$ and the outpatient sample of Project MATCH, ${ }^{21}$ two multi-site randomized clinical trials. Participants in the current analyses met criteria for AUD, received outpatient treatment, and constituted the subgroup of participants who were followed for up to 3 years post-treatment (COMBINE: $n=694$; MATCH: $n=806)$. Exclusion criteria for both studies included a current drug use disorder (other than nicotine or cannabis), a psychiatric disorder requiring medication, or an unstable medical condition.

COMBINE Study. Participants in the COMBINE Study $(n=$ 1383) met criteria for alcohol dependence based on the Diagnostic and Statistical Manual of Mental Disorders (DSM) (fourth edition) ${ }^{22}$ and engaged in heavy drinking prior to the baseline assessment. Participants were randomized into one of nine treatment cells and received 16 weeks of treatment via a $2 \times 2 \times 2$ design with eight of nine cells receiving (1) active naltrexone (100 mg/day) or placebo naltrexone, (2) active acamprosate $(3000 \mathrm{mg} /$ day $)$ or placebo acamprosate, and (3) medication management (MM) or combined behavioral intervention $(\mathrm{CBI})$ with $\mathrm{MM}$. The ninth cell received $\mathrm{CBI}$ and no pills.

Follow-up assessments were completed at the end of treatment (16 weeks post-randomization) and at 156 weeks postrandomization. Of the 1383 participants in the COMBINE trial, 874 patients from nine sites $(n=1144 ; 76.4 \%)$ consented to a longer-term follow-up, ${ }^{23,24}$ of whom 694 (79\%) provided data at the 3-year assessment.

Project MATCH. Participants in the outpatient sample of Project MATCH $(n=952)$ met the criteria for alcohol abuse (4.6\%) or alcohol dependence $(95.4 \%)$ based on the DSM (third edition-revised) ${ }^{25}$ and were actively drinking prior to

\begin{tabular}{|l|llll|}
\hline & \multicolumn{4}{|c|}{ World Health Organization Risk Levels (for males) } \\
& \multicolumn{1}{|c|}{ Low Risk } & \multicolumn{1}{c|}{ Medium Risk } & \multicolumn{1}{c|}{ High Risk } & \multicolumn{1}{c|}{ Very High Risk } \\
\hline Drinks per day (in grams) & 1 to $40 \mathrm{~g}$ & 41 to $60 \mathrm{~g}$ & 61 to $100 \mathrm{~g}$ & $101+\mathrm{g}$ \\
Drinks per day (approximate U.S. standard drinks, 14 g) & 0 to 3 drinks & 3 to <4 drinks & 4 to 7 drinks & 7 drinks \\
Drinks per week (approximate U.S. standard drinks, 14 g) & 0 to 20 drinks & 21 to 30 drinks & 31 to 50 drinks & $51+$ drinks \\
\hline
\end{tabular}

\begin{tabular}{|l|llll|}
\hline & \multicolumn{4}{|c|}{ World Health Organization Risk Levels (for females) } \\
& \multicolumn{1}{|c|}{ Low Risk } & \multicolumn{1}{|c|}{ Medium Risk } & \multicolumn{1}{c|}{ High Risk } & \multicolumn{1}{c|}{ Very High Risk } \\
\hline Drinks per day (in grams) & 1 to $20 \mathrm{~g}$ & 21 to $40 \mathrm{~g}$ & 41 to $60 \mathrm{~g}$ & $61+\mathrm{g}$ \\
Drinks per day (approximate U.S. standard drinks, 14 g) & 0 to 1 drinks & 2 to $<3$ drinks & 3 to $<4$ drinks & 4 drinks \\
Drinks per week (approximate U.S. standard drinks, 14 g) & 0 to $<10$ drinks & 10 to $<20$ drinks & 20 to 30 drinks & $31+$ drinks \\
\hline
\end{tabular}

Fig. 1 World Health Organization (WHO) risk drinking levels based on grams per day and approximate US standard drinks (14 g of pure alcohol) per day and per week. A 2-level reduction in WHO risk drinking level could include reductions from very high to medium, high to low, or low risk to abstinence. A 1-level reduction in WHO risk drinking level could include reductions from very high to high, high to medium, medium to low, or low risk abstinence. 
the baseline assessment. Participants were randomized to receive 12 weeks of treatment with cognitive behavioral therapy ${ }^{26}$ motivational enhancement therapy, ${ }^{27}$ or twelvestep facilitation. ${ }^{28}$

Follow-up assessments were completed at the end of treatment (12 weeks post-randomization) and at 156 weeks postrandomization. Of the 952 outpatients in MATCH, 806 patients (84.7\%) completed the 3-year follow-up assessment.

\section{Measures}

Alcohol Dependence Severity. The Alcohol Use Inventory $(\mathrm{AUI})^{29}$ was administered as a measure of dependence severity in MATCH. The Alcohol Dependence Scale (ADS), ${ }^{30}$ which is a modified version of the AUI, was administered in COMBINE. For the current study, we included the 23 items from the ADS and the AUI that were overlapping (Supplementary Table 1) assessing alcohol dependence severity in both studies ${ }^{31}(\alpha=0.85)$.

Alcohol Consumption. Standard drinks (14 g of absolute alcohol) were measured using calendar-based methods ${ }^{32,33}$ to document daily drinking amounts. WHO RDLs (abstinent, low risk, medium risk, high risk, very high risk) were calculated based on the average grams of alcohol consumed per day over 1-month time periods in the month prior to the baseline assessment, in the month prior to the end of treatment, and in the month prior to the 3-year follow-up. We then calculated reductions in RDLs from baseline to the end of treatment (predictor) and from baseline to the last month of the 3-year follow-up (outcome), defined by either a one-level reduction (i.e., reducing from very high to high, high to medium, medium to low, or low to abstinent) or a two-level reduction (i.e., reducing from very high to medium, high to low, or medium to abstinent). A one-level reduction was distinguished from no change or an increase in the WHO RDL from baseline to the treatment/follow-up months. A two-level reduction was distinguished from a one-level reduction, no change, or an increase in the WHO RDL from baseline to the treatment/ follow-up months. The one- and two-level reductions are not mutually exclusive categories, because anyone who achieved at least a two-level reduction also achieved a one-level reduction.

Functioning Outcomes at 3 Years Following Treatment. In COMBINE, mental health was assessed using the 6-item mental health subscale from the 12-item Short Form Health Survey (SF-12), ${ }^{34}$ with higher scores indicating better mental health functioning $(\alpha=0.82)$. Quality of life $(\mathrm{QoL})$ was assessed by a 25 -item version of the WHO QOL-BREF, ${ }^{35}$ a measure of general $\mathrm{QoL}^{36}$ that covers physical, psychological, environmental, and social health domains. Higher scores indicate better QoL.
In Project MATCH, drinking consequences were assessed with the Drinker Inventory of Consequences (DrInC) ${ }^{37}$ and psychosocial functioning was assessed using the Psychosocial Functioning Inventory (PFI). ${ }^{38}$ The $\operatorname{DrInC}^{37}$ is a 50 -item measure on which higher scores indicate more consequences $(\alpha=0.97)$. The $\mathrm{PFI}^{38}$ social behavior subscale includes 10 items that assess the frequency of problematic social behavior and social interactions. Higher scores on the PFI indicate better social functioning $(\alpha=0.83)$.

\section{Statistical Analysis}

The primary analyses examined the maintenance of one-level and two-level WHO RDL reductions and the association between such WHO RDLs in the last month of treatment and functioning at the 3-year follow-up. To test the maintenance/ stability of reductions, we examined the association between WHO RDL reductions achieved in the last month of treatment (predictor) and WHO RDL reductions achieved at the 3-year follow-up (outcomes) using logistic regression. Given both studies provided the same data for the logistic regression, we combined the data from the two studies to obtain a pooled estimate of the stability of WHO RDL reductions. We used linear regression to test the associations between WHO RDL reductions from baseline to the end of treatment (predictors) and functional outcomes at the 3-year follow-ups (outcomes). Because the two studies had different functional outcome measures at 3-year follow-ups, we conducted the linear regression analyses within each study separately. Unstandardized regression coefficients from the linear regression models can be interpreted as the differences in outcomes for patients with, versus without, oneor two-level RDL reductions, controlling for covariates. We also examined whether the severity of alcohol dependence at baseline moderated the relations between RDL reductions and functional outcomes.

All models were estimated with Mplus version $8.2^{39}$ using maximum likelihood with robust estimation to account for clustering within sites. ${ }^{40}$ Missing drinking and functional outcome data at the end of treatment and 3-year follow-up were accommodated via multiple imputation with 50 imputed datasets. ${ }^{41}$ Thus, we included all individuals who completed the 3-year follow-up assessments for both studies (MATCH $n=806$; COMBINE $n=694$ ). All variables examined in the current study were included in the imputation models. Consistent with prior analyses in the COMBINE Study data, ${ }^{4,9}$ we controlled for the following covariates in all analyses: age, sex, race, alcohol dependence severity, smoking status, and baseline WHO RDL.

Non-abstinent Reductions. To evaluate the effect on the results of individuals with total abstinence, the primary analyses were repeated with abstainers excluded from the models. 


\section{RESULTS}

\section{Descriptive Analyses}

Demographic characteristics and descriptive statistics for all measures from both studies are provided in Table 1. Most participants $(90.3 \%)$ were at the medium, high, or very high RDL at baseline, so a one-level reduction was possible for all participants and a two-level reduction was possible for nearly all participants. There were no significant demographic differences and no significant differences in baseline WHO RDLs between individuals based on whether they completed the 3year follow-up.

\section{Primary Analyses}

We used logistic regression to examine the odds of maintaining one- and two-level reductions in WHO RDLs at the 3-year follow-up. In a pooled analysis of data from both studies, $83.8 \%$ and $68.0 \%$ achieved at least one-level or two-level reductions, respectively, at the end of treatment, and $84.9 \%$ and $77.7 \%$ of those participants maintained at least one-level or two-level reductions, respectively, at the 3-year follow-up. Patients with RDL reductions at the end of treatment had a significantly higher odds of achieving the RDL reduction at the 3-year follow-up for both the one-level $(\mathrm{OR}=1.74 ; 95 \%$
CI: $1.59,1.88 ; p<0.001)$ and the two-level reductions $(\mathrm{OR}=$ $1.72 ; 95 \%$ CI: $1.60,1.85 ; p<0.001)$, indicating an ability to maintain/sustain the end of treatment RDL reductions at the 3year follow-up. The logistic regression models indicated no significant interaction effects with alcohol dependence severity in predicting the maintenance of RDL reductions at the 3year follow-up (all $p>0.08$ ).

Next, we used linear regression to examine the association between achieving at least one- and two-level reductions in WHO RDLs at the end of treatment and functional outcomes at the 3-year follow-up in each study separately. Full results from regression analyses are provided in Supplementary Table 2. Figure 2 shows functional outcomes among those who did and did not achieve drinking reductions in the last month of treatment, adjusted for covariates. Achieving at least a one-level RDL reduction in the last month of treatment, versus no change or an increase in RDL, was associated at the 3-year follow-up with significantly better mental health; greater environmental, social, psychological, and physical QoL; and significantly fewer drinking consequences; but no difference in psychosocial functioning. Achieving at least a two-level RDL reduction in the last month of treatment, versus a one-level RDL reduction, no change, or an increase in RDL, was associated with significantly better mental health and

Table 1 Demographics, WHO Risk Drinking Levels at Baseline, End of Treatment, and 3-Year Follow-up, and Functional Outcomes at Baseline and 3-Year Follow-ups by Study Among 3-Year Follow-up Completers

\begin{tabular}{|c|c|c|c|c|c|c|}
\hline & \multicolumn{3}{|c|}{ COMBINE $(n=694)$} & \multicolumn{3}{|c|}{ МАТСН $(n=806)$} \\
\hline & Baseline & End of treatment & $\begin{array}{l}\text { 3-year } \\
\text { follow-up }\end{array}$ & Baseline & End of treatment & $\begin{array}{l}\text { 3-year } \\
\text { follow-up }\end{array}$ \\
\hline \multicolumn{7}{|l|}{ Demographics } \\
\hline Age, mean (SD) & $44.9(10.3)$ & & & $38.6(10.7)$ & & \\
\hline Male $(\%)$ & $70.4 \%$ & & & $77.2 \%$ & & \\
\hline Non-Hispanic White (\%) & $78.3 \%$ & & & $79.4 \%$ & & \\
\hline Black/African American (\%) & $10.0 \%$ & & & $6.0 \%$ & & \\
\hline Hispanic $(\%)$ & $6.9 \%$ & & & $12.0 \%$ & & \\
\hline American Indian/Alaska Native (\%) & $1.2 \%$ & & & $1.7 \%$ & & \\
\hline Asian & $0.1 \%$ & & & $0.1 \%$ & & \\
\hline Multi-racial (\%) & $1.9 \%$ & & & $0.0 \%$ & & \\
\hline "Other" race (\%) & $1.5 \%$ & & & $0.7 \%$ & & \\
\hline \multicolumn{7}{|l|}{ WHO risk drinking levels } \\
\hline Abstinent, $N(\%)$ & $0(\%)$ & $260(37.6 \%)$ & $300(45.0 \%)$ & $0(\%)$ & $331(41.6 \%)$ & $309(38.3 \%)$ \\
\hline Low risk, $N(\%)$ & $17(2.4 \%)$ & $270(39.0 \%)$ & $161(24.1 \%)$ & $128(15.9 \%)$ & $302(37.9 \%)$ & $267(33.1 \%)$ \\
\hline Medium risk, $N(\%)$ & $51(7.3 \%)$ & $60(8.7 \%)$ & $63(9.4 \%)$ & $111(13.8 \%)$ & $55(6.9 \%)$ & $74(9.2 \%)$ \\
\hline High risk, $N(\%)$ & $143(20.6 \%)$ & $45(6.5 \%)$ & $71(10.6 \%)$ & $178(22.1 \%)$ & $43(5.4 \%)$ & $67(8.3 \%)$ \\
\hline Very high risk, $N(\%)$ & $483(69.6 \%)$ & $57(8.2 \%)$ & $72(10.8 \%)$ & $389(48.3 \%)$ & $65(8.2 \%)$ & $89(11.0 \%)$ \\
\hline At least 1 -level reduction from baseli & e, $N(\%)$ & $606(87.6 \%)$ & $548(82.2 \%)$ & & $641(80.5 \%)$ & $602(74.7 \%)$ \\
\hline At least 2-level reduction from baseli & $\mathrm{e}, N(\%)$ & $513(74.1 \%)$ & $454(68.1 \%)$ & & $498(62.6 \%)$ & $451(56.0 \%)$ \\
\hline \multicolumn{7}{|c|}{ Functional outcomes } \\
\hline SF-12 mental health, mean (SD) & $40.83(11.28)$ & $48.67(9.78)$ & $47.45(10.81)$ & & & \\
\hline Environmental QoL, mean (SD) & $29.33(5.55)$ & $31.00(5.57)$ & $31.38(5.29)$ & & & \\
\hline Social QoL, mean (SD) & $9.76(2.64)$ & $10.81(2.57)$ & $10.90(2.57)$ & & & \\
\hline Psychological QoL, mean (SD) & $20.79(4.00)$ & $22.55(4.26)$ & $22.80(4.06)$ & & & \\
\hline Physical QoL, mean (SD) & $27.02(4.34)$ & $29.02(4.33)$ & $28.33(4.66)$ & & & \\
\hline DrInC scores, mean (SD) & & & & $46.73(22.23)$ & $33.55(25.08)$ & $33.11(24.89)$ \\
\hline PFI social behavior, mean (SD) & & & & $3.22(0.49)$ & $3.41(0.47)$ & $3.43(0.47)$ \\
\hline
\end{tabular}

All descriptive statistics are observed with no imputation for missing data. SF-12, Short Form Health Survey, where higher scores indicate better mental health; QoL, World Health Organization Quality of Life Scale, where higher scores indicate better quality of life; DrInC, Drinker Inventory of Consequences Scale, where higher scores indicate more alcohol-related consequences; PFI, Psychosocial Functioning Inventory, where higher scores indicate better psychosocial functioning 


\section{SF-12 Mental Health (COMBINE)}
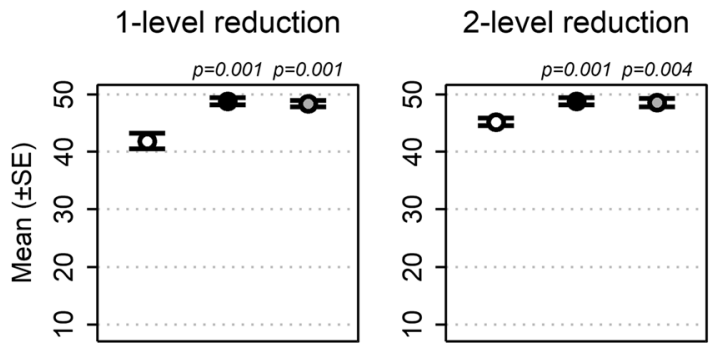

Environmental QoL (COMBINE)

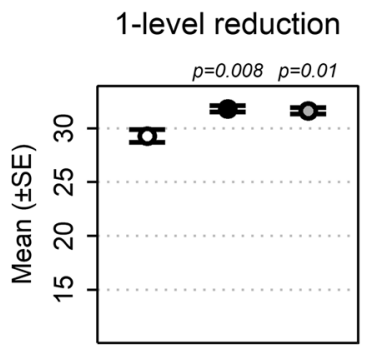

2-level reduction

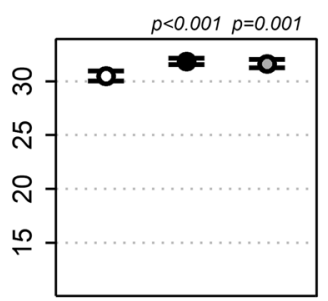

Psychological QoL (COMBINE)

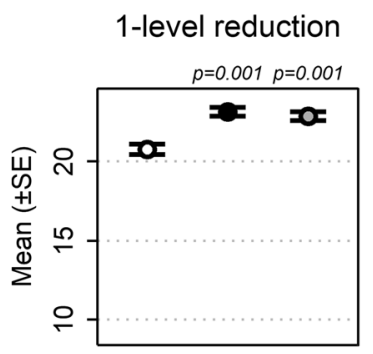

2-level reduction

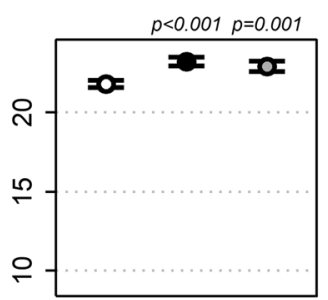

- No drinking risk level reduction
- Drinking risk level reduction
- Drinking risk level reduction (not abstinent)

Social QoL (COMBINE)

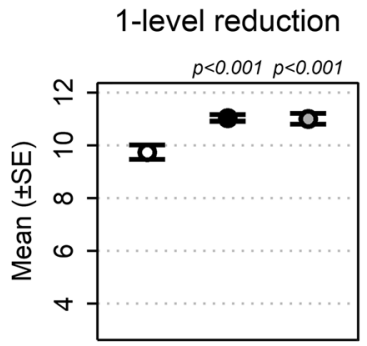

2-level reduction

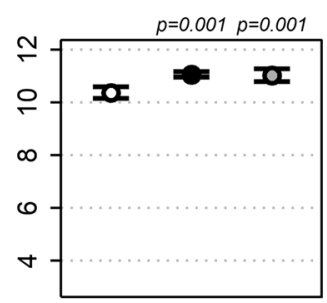

Drinker Inventory of Consequences (DrInC, MATCH) (lower is better) 1-level reduction

$p<0.001 p=0.008$

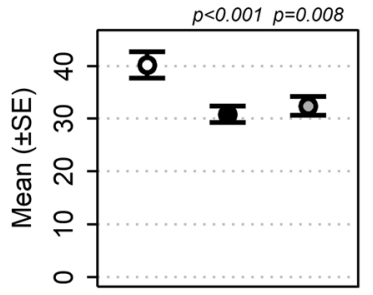

2-level reduction

$p<0.001 \quad p=0.01$

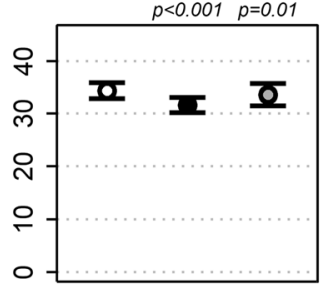

Psychosocial Functioning Inventory (PFI, MATCH)

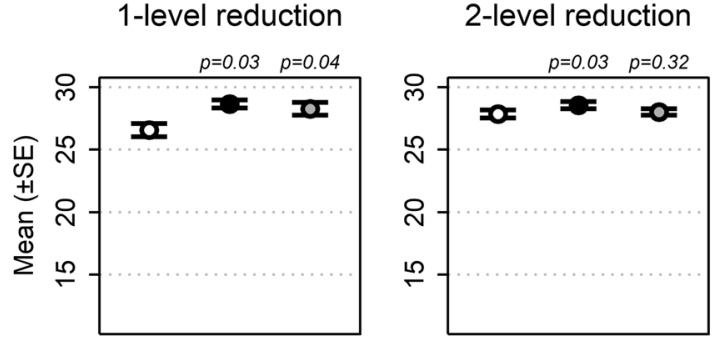

Fig. 2 Relative difference in functional outcomes assessed at the 3-year follow-up based on risk drinking level reductions achieved at the end of initial treatment, adjusted for other covariates in linear regression analyses. Functional outcomes at the 3-year follow-up based on achieving at least a one- or two-level reduction at the end of treatment (versus achieving no change or increase in drinking risk), controlling for covariates, with $p$ values from linear regression analyses comparing the one- and two-level reductions to the reference group. $Y$-axis scaling based on the lower bound for each measure. The reference group for the one-level reduction was no change or an increase in the WHO RDL from baseline to the last month of treatment. The reference group for the two-level reduction was the one-level reduction, no change, or increase in the WHO RDL from baseline to the last month of treatment. SF-12, Short Form Health Survey, where higher scores indicate better mental health; QoL,

World Health Organization (WHO) QOL-BREF scale, where higher scores indicate better quality of life; DrInC, Drinker Inventory of Consequences Scale, where higher scores indicate more alcohol-related consequences; PFI, Psychosocial Functioning Inventory, where higher scores indicate better psychosocial functioning. 
(a) One-level RDL reduction predicting social quality of life (higher scores indicate greater quality of life)

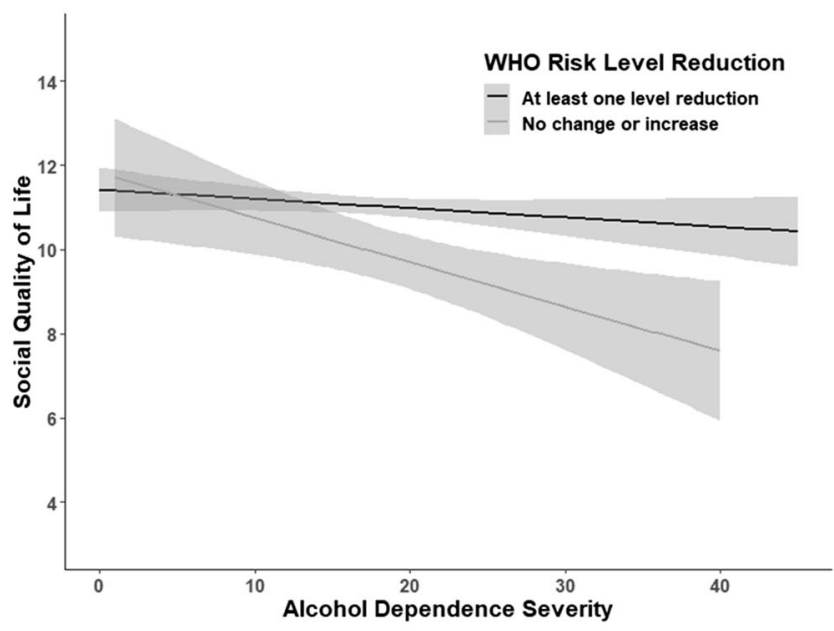

(b) Two-level RDL reduction predicting social quality of life (higher scores indicate greater quality of life)

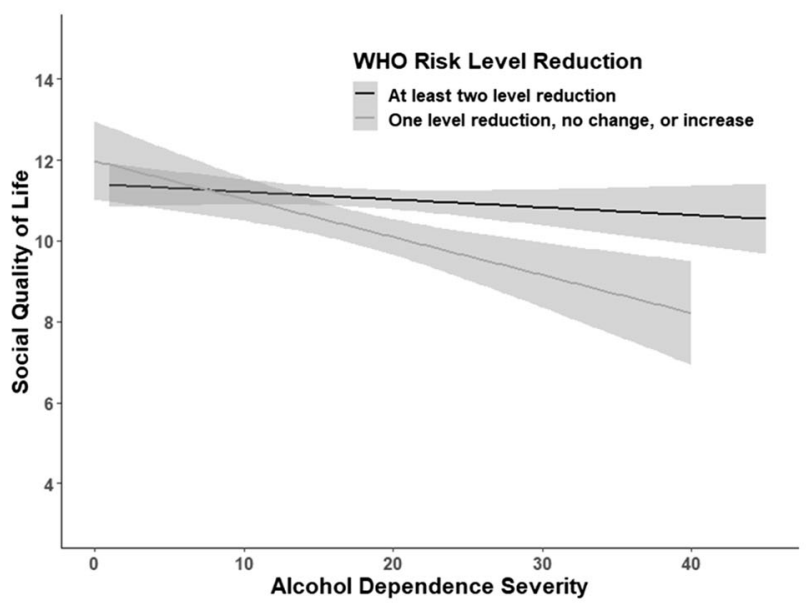

(c) Two-level RDL reduction predicting physical quality of life (higher scores indicate greater quality of life)

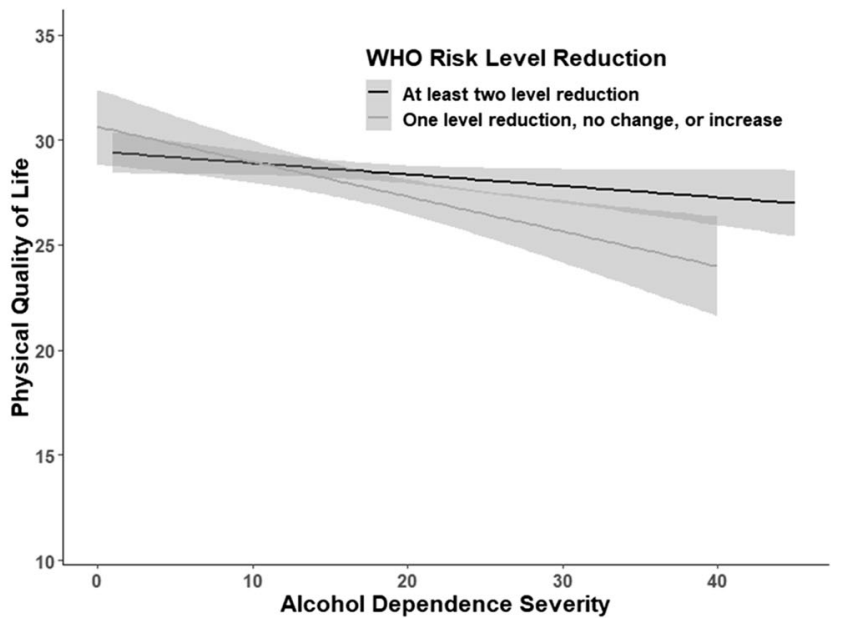

Fig. 3 Interaction between baseline alcohol dependence severity and WHO risk drinking level reduction achieved at the end of treatment in predicting quality of life outcomes at the 3-year follow-up assessment in COMBINE. a One-level RDL reduction predicting social quality of life (higher scores indicate greater quality of life). b Two-level RDL reduction predicting social quality of life (higher scores indicate greater quality of life). $\mathrm{c}$ Two-level RDL reduction predicting physical quality of life (higher scores indicate greater quality of life). Quality of life (QoL) was assessed by the World

Health Organization (WHO) QOL-BREF scale, a measure of general QoL that covers physical, psychological, environmental, and social health domains. Higher scores indicate better QoL and $Y$-axis ranges based on observed data values.

QoL, significantly fewer drinking consequences, and significantly better psychosocial functioning.

The linear regression models indicated significant interaction effects between dependence severity and one- and twolevel RDL reductions in predicting social QoL and between dependence severity and two-level reductions in predicting physical QoL (Supplementary Table 2). The interaction effect showed that reductions in the WHO RDL conferred the greatest benefits in social and physical QoL at the 3-year follow-up among individuals higher in dependence severity at baseline (Fig. 3 panels a-c), perhaps because their quality of life was lower prior to treatment.

\section{Non-abstinent Drinking Reductions}

Next, we re-estimated all models with abstainers excluded from the analysis $(N=432$ for COMBINE and $N=461$ for MATCH). Reductions in WHO RDLs, short of total abstinence, in the last month of treatment were significantly associated with one- and two-level reductions in WHO RDLs at the 3-year follow-up. In a pooled analysis of data from both studies, $73.1 \%$ and $54.4 \%$ of non-abstainers achieved at least one-level or two-level reductions, respectively, at the end of treatment, and $82.6 \%$ and $73.0 \%$ of those participants maintained at least one-level or two-level reductions, respectively, at the 3-year follow-up (odds of risk drinking reduction at 3 years predicted by risk drinking reduction at end of treatment $=1.70$ for a one-level reduction $(95 \%$ CI: $1.53,1.87 ; p$ $<0.001)$ and 1.57 for a two-level reduction (95\% CI: 1.41 , $1.72 ; p<0.001)$ ).

Regression analyses that removed abstainers from the analyses yielded findings similar to the prior analyses (Fig. 2 and Supplementary Table 2), although the effects on functional outcomes were smaller and the effects of two-level reductions on physical QoL and psychosocial functioning were not significant with abstainers excluded. There were also significant interaction effects between dependence severity and one- and two-level RDL reductions in predicting social QoL, and between dependence severity and two-level RDL reductions in predicting mental health (Supplementary Table 2). Nonabstinent drinking reductions during the last month of treatment conferred the greatest benefits in social QoL and mental health at the 3-year follow-up for individuals highest in dependence severity (Supplementary Fig. 1 panels a-c). 


\section{DISCUSSION}

We examined whether reductions in WHO RDLs were maintained and associated with improvements in how patients feel and function, both physically and mentally, up to 3 years following treatment in two large alcohol clinical trials. The one- and two-level WHO risk reductions at the end of treatment were largely maintained and were associated with significantly better health functioning at up to 3 years following treatment. A large majority (76-87\%) of individuals who achieved one- or two-level WHO risk reductions by the last month of treatment maintained those reductions at the 3-year follow-up. Moreover, individuals who achieved one- or twolevel reductions in WHO RDLs by the last month of treatment had significantly better mental health and physical QoL and fewer drinking consequences at the 3-year follow-up than those who failed to achieve such reductions.

Additional analyses that excluded abstainers and tested interactions with alcohol dependence severity were largely consistent with the primary findings, with a few exceptions. Removing abstainers from the models rendered the effects of a two-level WHO RDL reduction on physical QoL and psychosocial functioning non-significant; although the size of the effects was similar. Participants with the most severe alcohol dependence had the most to gain in social and physical QoL and mental health by reducing their drinking during treatment, even short of full abstinence.

The results from the current study are consistent with recent studies showing that WHO RDL reductions are maintained for up to 1 year following treatment ${ }^{7}$ and significantly associated with better patient functioning. ${ }^{4,5}$ The current study extends these findings by providing evidence that WHO RDL reductions are maintained for up to 3 years following treatment and associated with concomitant improvements in functioning. Consistent with recent reviews on drinking reductions, ${ }^{3,10,42}$ the current findings indicate that among treatment seekers with AUD, reductions in drinking are achievable, maintained, and associated with long-term improvements in functioning.

The current study depended upon available data and the inclusion/exclusion criteria used in the COMBINE Study and Project MATCH. Both studies included treatment seekers with AUD who received some form of AUD treatment (psychosocial or medications) and excluded individuals with a drug use disorder or severe psychiatric and medical conditions. Whether these findings would generalize to patients with more or less severe presentations, to patients with comorbid substance use or severe psychiatric or medical disorders, or to non-treatment seekers cannot be determined from the current study. Yet, data from a large general US adult sample have shown that reductions in WHO RDLs over a 3-year period correlate with reduced odds of current alcohol dependence, drug use disorders, depression, and anxiety disorders. ${ }^{5,8,43}$ Future studies should recruit more diverse patient populations and extend the current analyses by examining the maintenance of WHO RDL reductions in association with more objective markers of how patients feel and function, including physical health measures, biomarkers of alcohol consumption and organ function, medical expenditures, employment data, and functioning as reported by family/friends.

Consistent with a growing body of findings from other reports $^{3-5,9,42-44}$ and clinical guidelines, ${ }^{45-47}$ our results suggest that reduced drinking, as measured by the WHO RDL reduction metric, is associated with good clinical outcomes and can be sustained over time. Some clinicians might prefer suggesting at least a 1-level reduction for patients, whereas others might prefer suggesting at least a 2-level reduction. The current study provides evidence that either recommendation is likely to be sustained and associated with meaningful improvement in how patients feel and function. This information should be disseminated to inform clinical providers and the public. The information could encourage individuals with AUD who are reluctant to participate in abstinence-focused treatment to set goals with their clinical providers that are short of abstinence yet capable of providing substantial clinical benefit.

Contributors: In addition to the authors, the following individuals are or were members of the Alcohol Clinical Trials Initiative (ACTIVE) Workgroup and provided intellectual input during attendance at Workgroup meetings: Raye Z. Litten, Ph.D.; Daniel E. Falk, Ph.D.; Joanne Fertig, Ph.D.; and Megan Ryan, Ph.D., National Institute on Alcohol Abuse and Alcoholism; Tanya Ramey, M.D., Ph.D., and David McCann, Ph.D., National Institute on Drug Abuse; Didier Meulien, M.D., Lundbeck SAS; Anne Andorn, M.D., and Jay Graham, M.D., Indivior; Roger Meyer, M.D., Best Practice Project Management, Inc.; Henri-Jean Aubin, M.D., Paris-Sud Medical School; Charles O'Brien, M.D., Ph.D., University of Pennsylvania; Bernard Silverman, M.D., Alkermes, Inc.; Francoise Trinquet, M.D., Ethypharm; Benjamin Zakine, M.D., Ethypharm. Lindsay Snyder and Sarah Timm (ASCP) provided important administrative support to the ACTIVE workgroup. Raye Z. Litten, Ph.D., and Daniel E. Falk, Ph.D., National Institute on Alcohol Abuse and Alcoholism, provided feedback on earlier drafts of this manuscript.

Corresponding Author: Katie Witkiewitz, PhD; Department of Psychology, University of New Mexico, MSC 03-2220, Albuquerque, NM 87131, USA (e-mail: katiew@unm.edu).

Funding This study was funded by the U.S. National Institute on Alcohol Abuse and Alcoholism (RO1AA022328), Bethesda, MD, USA, to Dr. Witkiewitz.

Data Availability The original datasets for Project MATCH and COMBINE are available from the National Institute on Alcohol Abuse and Alcoholism (http://www.niaaa.nih.gov).

\section{Compliance with Ethical Standards:}

Conflict of Interest: Dr. Kranzler is named as an inventor on PCT patent application \#15/878,640 entitled "Genotype-guided dosing of opioid agonists," filed January 24, 2018. Dr. Hasin is principal investigator of a study funded by inVentiv Health Consulting that combines support from Actavis, Inc.; Endo Pharmaceuticals; Janssen Pharmaceuticals, Inc.; Mallinckrodt, LLC; Pfizer, Inc.; Purdue Pharma, L.P.; Rhodes Pharmaceuticals, L.P.; Roxane Laboratories, Inc.; and Zogenix, Inc. Dr. O'Malley reports being a consultant or an advisory board member of Alkermes, Indivior, Mitsubishi Tanabe, and Opiant, and a NIDA Clinical Trials Network DSMB member with honorarium from the Emmes Corporation and non-financial support from Amygdala Neurosciences, Astra Zeneca, and Novartis. Dr. Anton has been a consultant in recent past for Insys, Allergan, and Life Epigenetics, has received honorarium from Alkermes for grant reviews and advisory board 
activities, and currently has grant funding from Laboratorio Farmaceutico CT. Dr. Hallgren reports personal fees from Pear Therapeutics, outside the submitted work. Drs. Witkiewitz, Kranzler, Mann, Hasin, O'Malley, Aldridge, Zarkin, and Anton are members of or have participated in meetings sponsored by the American Society of Clinical Psychopharmacology's Alcohol Clinical Trials Initiative (ACTIVE Group), which over the time that this paper was developed was supported by Alkermes, Amygdala Neurosciences, Arbor Pharmaceuticals, Ethypharm, Indivior, Lundbeck, Mitsubishi, and Otsuka.

Disclaimer: The funders had no role in the design, analysis, interpretation, or publication of this study.

Open Access This article is licensed under a Creative Commons Attribution 4.0 International License, which permits use, sharing, adaptation, distribution and reproduction in any medium or format, as long as you give appropriate credit to the original author(s) and the source, provide a link to the Creative Commons licence, and indicate if changes were made. The images or other third party material in this article are included in the article's Creative Commons licence, unless indicated otherwise in a credit line to the material. If material is not included in the article's Creative Commons licence and your intended use is not permitted by statutory regulation or exceeds the permitted use, you will need to obtain permission directly from the copyright holder. To view a copy of this licence, visit http://creativecommons. org/licenses/by/4.0/.

\section{REFERENCES}

1. Witkiewitz $\mathbf{K}$, Litten $\mathbf{R Z}$, Leggio $\mathbf{L}$. Advances in the science and treatment of alcohol use disorder. Sci Adv. 2019;5(9):eaax4043. https:// doi.org/10.1126/sciadv.aax4043

2. Park-Lee E, Lipari RN, Hedden SL, Kroutil LA, Porter J. Receipt of Services for Substance Use and Mental Health Issues among Adults: Results from the 2016 National Survey on Drug Use and Health.; 2017. https://www.samhsa.gov/data/sites/default/files/NSDUH-DR-FFR2 2016/NSDUH-DR-FFR2-2016.htm.

3. Mann K, Aubin H-J, Witkiewitz K. Reduced drinking in alcohol dependence treatment, What Is the evidence? Eur Addict Res 2017;23(5):219-230. https://doi.org/10.1159/000481348

4. Witkiewitz K, Hallgren KA, Kranzler HR, et al. Clinical validation of reduced alcohol consumption after treatment for alcohol dependence using the World Health Organization risk drinking levels. Alcohol Clin Exp Res 2017;41(1):179-186. https://doi.org/10.1111/ACER. 13272

5. Hasin DS, Wall M, Witkiewitz $\mathbf{K}$, et al. Change in non-abstinent WHO drinking risk levels and alcohol dependence: a 3 year follow-up study in the US general population. Lancet Psychiatry 2017;4(6):469-476. https://doi.org/10.1016/S2215-0366(17)30130-X

6. Knox J, Wall M. Witkiewitz $\mathbf{K}$, et al. Reduction in nonabstinent WHO drinking risk levels and change in risk for liver disease and positive AUDIT-C scores: Prospective 3-year follow-up results in the U.S. general population. Alcohol Clin Exp Res 2018;42(11):2256-2265. https://doi. org/10.1111/acer.13884

7. Witkiewitz K, Falk DE, Litten RZ, et al. Maintenance of World Health Organization risk drinking level reductions and posttreatment functioning following a large alcohol use disorder clinical trial. Alcohol Clin Exp Res 2019;43(5):979-987. https://doi.org/10.1111/acer. 14018

8. $\mathbf{K n o x} \mathbf{J}$, Wall M. Witkiewitz $\mathbf{K}$, et al. Reduction in non-abstinent World Health Organization (WHO) drinking risk levels and drug use disorders: 3-year follow-up results in the US general population. Drug Alcohol Depend 2019;201:16-22. https://doi.org/10.1016/j.drugalcdep.2019. 03.020

9. Witkiewitz K, Kranzler HR, Hallgren KA, et al. Drinking risk level reductions associated with improvements in physical health and quality of life among individuals with alcohol use disorder. Alcohol Clin Exp Res 2018;42(12):2453-2465. https://doi.org/10.1111/acer.13897

10. van Amsterdam $\mathbf{J}$, van den Brink $\mathbf{W}$. Reduced-risk drinking as a viable treatment goal in problematic alcohol use and alcohol dependence. $J$ Psychopharmacol 2013;27(11):987-997. https://doi.org/10.1177/ 0269881113495320

11. Food and Drug Administration. Alcoholism: Developing Drugs for Treat ment. Silver Spring, MD; 2015. http://www.regulations.gov/ \#!documentDetail;D=FDA-2015-D-0152-0001.
12. European Medicines Agency. Guideline on the Development of Medicinal Products for the Treatment of Alcohol Dependence. Amsterdam: European Medicines Agency; 2010.

13. World Health Organization (WHO). International Guide for Monitoring Alcohol Consumption and Related Harm. Geneva: World Health Organization; 2000.

14. Vaillant GE. A 60-year follow-up of alcoholic men. Addiction. 2003;98(8):1043-1051. http://www.ncbi.nlm.nih.gov/pubmed/ 12873238. Accessed June 11, 2016

15. Gual A, Bravo F, Lligona A, Colom J. Treatment for alcohol dependence in Catalonia: Health outcomes and stability of drinking patterns over 20 years in 850 patients. Alcohol Alcohol 2009;44(4):409-415. https://doi. org/10.1093/alcalc/agp032

16. Subbaraman MS, Witbrodt J. Differences between abstinent and nonabstinent individuals in recovery from alcohol use disorders. Addict Behav 2014;39(12):1730-1735. https://doi.org/10.1016/j.addbeh.2014. 07.010

17. Berglund $\mathbf{K J}$, Rauwolf $\mathbf{K K}$, Berggren U, Balldin J, Fahlke C. Outcome in relation to drinking goals in alcohol-dependent individuals: A follow-up study 2.5 and 5 years after treatment entry. Alcohol Alcohol 2019;54(4):439-445. https://doi.org/10.1093/ alcalc/agz042

18. Sobell MB, Sobell LC. Controlled drinking after 25 years: how important was the great debate? Addiction. 1995:90(9):1149-1153; discussion 115777. http://www.ncbi.nlm.nih.gov/pubmed/7580815. Accessed June 11, 2016.

19. Davis AK, Rosenberg H. Acceptance of non-abstinence goals by addiction professionals in the United States. Psychol Addict Behav 2013;27(4):1102-1109. https://doi.org/10.1037/a0030563

20. Anton RF, O'Malley SS, Ciraulo DA, et al. Combined pharmacotherapies and behavioral interventions for alcohol dependence: the COMBINE study: a randomized controlled trial. JAMA. 2006;295(17):2003-2017. https://doi.org/10.1001/jama.295.17.2003

21. Project MATCH Research Group. Matching Alcoholism Treatments to Client Heterogeneity: Project MATCH posttreatment drinking outcomes. $J$ Stud Alcohol 1997;58(1):7-29. http://www.ncbi.nlm.nih.gov/pubmed/ 8979210. Accessed September 27, 2013.

22. American Psychiatric Association. Diagnostic and Statistical Manual of Mental Disorders (4th Ed.; DSM-IV). 4th ed. Washington, DC, DC: Author; 1994

23. Zarkin GA, Bray JW, Aldridge A, et al. The effect of alcohol treatment on social costs of alcohol dependence. Med Care 2010;48(5):396-401. https://doi.org/10.1097/MLR.0b013e3181d68859

24. Zarkin GA, Bray JW, Aldridge A, et al. Cost and cost-effectiveness of the COMBINE study in alcohol-dependent patients. Arch Gen Psychiatry 2008;65(10):1214-1221. https://doi.org/10.1001/archpsyc.65.10.1214

25. American Psychiatric Association. Diagnostic and Statistical Manual of Mental Disorders (3rd Ed., Rev.; DSM-III-R). 3rd ed., r. Washington, DC, DC: Author.; 1987.

26. Kadden RM, Carroll KM, Donovan DM, et al. Cognitive-Behavioral Coping Skills Therapy Manual. Rockville, MD: National Institute on Alcohol Abuse and Alcoholism; 1995. https://pubs.niaaa.nih.gov/publications/MATCHSeries3/Project MATCH Vol_3.pdf.

27. Miller WR, Zweben A, DiClemente CC, Rychtarik RG. Motivation Enhancement Therapy Manual. Rockville: National Institute on Alcohol Abuse and Alcoholism; 1994.

28. Nowinski J, Baker S, Carroll KM. Twelve Step Facilitation Therapy Manual. Rockville: National Institute on Alcohol Abuse and Alcoholism; 1995.

29. Horn JL, Wanberg KW, Foster FM. Guide to the Alcohol Use Inventory (AUI). Minneapolis: National Computer Systems; 1990.

30. Skinner HA, Horn JL. Alcohol Dependence Scale (ADS) User's Guide. Toronto: Addiction Research Foundation; 1984.

31. Witkiewitz K, Hallgren KA, O'Sickey AJ, Roos CR, Maisto SA Reproducibility and differential item functioning of the alcohol dependence syndrome construct across four alcohol treatment studies: An integrative data analysis. Drug Alcohol Depend 2016;158:86-93. https://doi.org/10.1016/j.drugalcdep.2015.11.001

32. Miller WR. Form 90: A Structured Assessment Interview for Drinking and Related Behaviors. Project MA. Bethesda: National Institute on Alcohol Abuse and Alcoholism; 1996.

33. Sobell LC, Sobell MB. Timeline Follow-Back: A Technique for Assessing Self-Reported Alcohol Consumption. (Litten RZ, Allen JP, eds.). Totowa: Human Press; 1992. http://search.proquest.com/ psycinfo/docview / 618232357 / 13607B0066F5980EE4 1/ 1? accountid=14902. 
34. Ware JE, Kosinski M, Keller SD. A 12-Item Short-Form Health Survey: construction of scales and preliminary tests of reliability and validity. Med Care 1996;34(3):220-233. http://www.ncbi.nlm.nih.gov/pubmed/ 8628042. Accessed July 14, 2014.

35. World Health Organization. World Health Organization Quality of Life. Geneva: World Health Organization; 1998.

36. Kirouac M, Stein ER, Pearson MR, Witkiewitz K. Viability of the World Health Organization quality of life measure to assess changes in quality of life following treatment for alcohol use disorder. Qual Life Res 2017;26(11):2987-2997. https://doi.org/10.1007/s11136-017-1631-4

37. Miller WR, Tonigan JS, Longabaugh $\mathbf{R}$. The Drinker Inventory of Consequences (DrInC). Project MA. Bethesda, MD: National Institute on Alcohol Abuse and Alcoholism; 1995.

38. Feragne MA, Longabaugh R, Stevenson JF. The Psychosocial Functioning Inventory. Eval Health Prof 1983;6(1):25-48. https://doi.org/10. $1177 / 016327878300600102$

39. Muthén LK, Muthén BO. Mplus users guide (Version 8). 2019.

40. Yuan K-H, Bentler PM. Finite normal mixture SEM analysis by fitting multiple conventional SEM models. Sociol Methodol 2010;40(1):191-245. https://doi.org/10.1111/j.1467-9531.2010.01224.x

41. Hallgren KA, Witkiewitz $\mathbf{K}$. Missing data in alcohol clinical trials: a comparison of methods. Alcohol Clin Exp Res 2013;37(12):2152-2160. https://doi.org/10.1111/acer. 12205

42. Charlet $\mathbf{K}$, Heinz A. Harm reduction - a systematic review on effects of alcohol reduction on physical and mental symptoms. Addict Biol 2017;22(5):1119-1159. https://doi.org/10.1111/adb.12414
43. Knox J, Scodes J, Wall M, et al. Reduction in non-abstinent WHO drinking risk levels and depression/anxiety disorders: 3-year follow-up results in the US general population. Drug Alcohol Depend 2019; 197:228235. https://doi.org/10.1016/j.drugalcdep.2019.01.009

44. Falk DE, O'Malley SS, Witkiewitz $\mathbf{K}$, et al. Evaluation of drinking risk levels as outcomes in alcohol pharmacotherapy trials. JAMA Psychiatry 2019;76(4):374. https://doi.org/10.1001/jamapsychiatry.2018.3079

45. National Institute for Health and Care Excellence. Alcohol-Use Disorders: Diagnosis, Assessment and Management of Harmful Drinking (High-Risk Drinking) and Alcohol Dependence (NICE Clinical Guideline No. CG115).; 2011. https://www.nice.org.uk/guidance/cg115.

46. National Institute on Alcohol Abuse and Alcoholism. Helping Patients Who Drink Too Much: A Clinician's Guide. Bethesda: National Institutes of Health; 2005.

47. Mann K, Batra A, Fauth-Bühler M, Hoch E. German guidelines on screening, diagnosis and treatment of alcohol use disorders. Eur Addict Res 2017;23(1):45-60. https://doi.org/10.1159/000455841

Publisher's Note Springer Nature remains neutral with regard to jurisdictional claims in published maps and institutional affiliations. 